\title{
SHAPE FROM LAMBERTIAN PHOTOMETRIC FLOW FIELDS
}

\author{
Lawrence B. Wolff ${ }^{1}$ \\ Computer Science Department \\ Columbia University \\ New York. N.Y. 10027 \\ Columbia University Technical Report CS-396-88 \\ October 1988
}

\begin{abstract}
A new idea for the analysis of shape from reflectance maps is introduced in this paper. It is shown that local surface orientation and curvature constraints can be obtained at points on a smooth surface by computing the instantaneous rate of change of reflected scene radiance caused by angular variations in illumination geometry. The resulting instantaneous changes in image irradiance values across an optic sensing array of pixels constitute what is termed a photometric flow field. Unlike optic flow fields which are instantaneous changes in position across an optic array of pixels caused by relative motion, there is no correspondence problem with respect to obtaining the instantaneous change in image irradiance values between successive image frames. This is because the object and camera remain static relative to one another as the illumination geometry changes.

There are a number of advantages to using photometric flow fields. One advantage is that local surface orientation and curvature at a point on a smooth surface can be uniquely determined by only slightly varying the incident orientation of an illuminator within a small local neighborhood about a specific incident orientation. Robot manipulators and rotation/positioning jigs can be accurately varied within small ranges of motion. Conventional implementation of photometric stereo requires the use of three vastly different incident orientations of an illuminator requiring either much calibration and/or gross and inaccurate robot arm motions. Another advantage of using photometric flow fields is the duality that exists between determining unknown local surface orientation from a known incident illuminator orientation and determining an unknown incident illuminator orientation from a known local sudface orientation. The equations for photometric flow fields allow the quantitative determination of the incident orientation of an illuminator from an object having a known calibrated surface orientation. Computer simulations will be shown depicting photometric flow fields on a Lambertian sphere. Simulations will be shown depicting how photometric flow fields quantitatively determine local surface orientation from a known incident orientation of an illuminator as well as determining incident illuminator orientation from a known local surface orientation.
\end{abstract}

\footnotetext{
${ }^{1}$ This work was supported in part by ARPA grant \#N00039-84-C-0165 and NSF grant IRI-88-00370. This work was supported in part by an IBM Graduate Fellowship A ward.
} 


\section{INTRODUCTION}

Vision techniques that rely upon feature information from discontinuities in an image (e.g. edges,corners and lines) quickly breakdown in regions containing smooth surfaces where gray level intensities are generally smoothly varying. It is in these regions of an image that modeling the reflectance properties of an object is most useful. The notion of the reflectance map presented in [Horn 1977] is a convenient way of directly relating reflected scene radiance to the surface orientation of a given object material. Such a relation, if physically accurate, can be very useful in obtaining shape information about smooth objects in an image. Two techniques which have paved the way for many other techniques making use of reflectance maps are the method of shape from shading presented in [Horn 1975] and the method of photometric stereo presented in [Woodham 1978]. Both of these methods make the simplifying assumption that the reflectance maps for an object material are Lambertian. A shape from shading method based on local analysis is presented in [Pentland 1984]. Implementation of photometric stereo assuming the presence of specular reflection has been reported in [Ikeuchi 1981] and [Ikeuchi et al. 1986].

A major problem in obtaining local surface orientation at a point on a smooth surface from a single view is the nonuniqueness of surface orientations consistent with the reflected radiance measured from the point. This is because for a given illuminator orientation the reflectance map supplies a single equation constraint between reflected radiance and local surface orientation. By varying the incident orientation of the illuminator, photometric stereo methods obtain additional equation constraints. This is because the reflectance map is dependent on illuminator orientation. However, to get additional equation constraints that are independent in the presence of noise, large variations in incident orientation of the illuminator are required.

This paper introduces another approach to using reflectance maps to obtain relations between local surface orientation and empirically measured photometric quantities. This new approach involves measuring how reflected radiance varies at a point on a smooth surface by varying the angular orientation of the illuminator. By measuring how reflected radiance varies as the illuminator moves in both linearly independent angular directions, two independent equations which constrain local surface orientation are obtained. These newly obtained relations enable the unique determination of local surface orientation from a single view, and angular displacement of a single illuminator within a small neighborhood about a specific incident orientation.

The difference of the reflected radiance at a point on a smooth surface between two slightly displaced incident orientations of an illuminator, divided by the angular displacement, is an approximation to the directional derivative of the reflectance function in the direction of the displacement of the illuminator. This photometric quantity measured at all pixels in an image corresponding to illuminated object points constitutes a photometric flow field with respect to the angular displacement of the illuminator in a given direction. The actual directional derivative of a reflectance function in this direction is also a function of local surface orientation. In the case of a Lambertian reflectance function in gradient space representation. the directional derivatives in both angular components of illuminator orientation have the same functional form with respect to gradient space variables $p$ and $q$ representing local surface orientation. These directional derivatives can take on both positive and negative values at points on a surface which are illuminated. Positive values denote an increase in reflected radiance at a point as the light source moves in a given angular direction, and negative values a corresponding decrease in reflected radiance. Except for the fact that the directional derivatives can take on both negative and positive values. they are also functions which are continuously differentiable in variables representing surface orientation and can serve as functions equivalent to reflectance functions with respect to any shape from shading and photometric stereo algorithm. The 
advantage of evaluating two independent photometric flow fields at a point on a smooth surface should be clear. Other than the surface orientation constraint equation provided by the reflectance function itself, two additional surface orientation contraints are provided from the photometric flow fields.

There is also a big additional advantage to using photometric flow fields which is not possible from conventional use of photometric stereo. This is the duality that exists between the determination of local surface orientation using the known incident orientation of a single illuminator, and the determination of the incident illuminator orientation from a known local surface orientation. Since the evaluation of both independent photometric flow fields at a point on a surface correspond to the same incident illuminator orientation, a known local orientation at that point can be used to determine the incident orientation of the illuminator. This will be demonstrated below.

The reflectance map assumed in this paper is Lambertian. Not only is this reflectance map simple to work with, but in light of recent work which has been successful in isolating the diffuse component of reflection from a variety of material surfaces, Lambertian reflectance is realistic. Reported in [Shafer 1985] and [Klinker et al. 1987] are techniques that separate diffuse and specular components of reflection based upon color analysis. Reported in [Wolff 1988] is an approach to separating diffuse and specular components of reflection using a polarizing filter which isolates polarization components. Since the diffuse component of reflection is Lambertian in nature, even on rough surfaces, Lambertian photometric flow fields can be analyzed on the diffuse component image.

\section{LAMBERTIAN PHOTOMETRIC FLOW FIELDS}

Using gradient space representation, the reflectance map for a Lambertian surface is given by the function

$$
R(p, q)=\frac{p \cos \tau \sin \sigma+q \sin \tau \sin \sigma+\cos \sigma}{\sqrt{p^{2}+q^{2}+1}}
$$

where $(p, q)$ are the gradient space coordinates for the surface orientation, and $\sigma$ and $\tau$ represent the slant and tilt angles for the illuminator (see figure 1). For a given measured value of the reflected radiance, equation 1 becomes the equation for a conic section curve in gradient space. This is referred to in photometric stereo techniques as an isoreflectance curve because it gives all possible gradient space representations of surface orientation that are consistent with the measured reflected radiance value.

Suppose now that the incident orientation of the illuminator is shifted in the slant angle $\sigma$ by a small amount $\Delta \sigma$. The instantaneous rate of change of the reflected radiance in the direction of $\Delta \sigma$ can be approximated by the quantity

$$
\frac{\frac{p \cos \tau \sin (\sigma+\Delta \sigma)+g \sin \tau \sin (\sigma+\Delta \sigma)+\cos (\sigma+\Delta \sigma)}{\sqrt{p^{2}+q^{2}+1}}-\frac{p \cos \tau \sin \sigma+q \sin \tau \sin \sigma+\cos \sigma}{\sqrt{p^{2}+q^{2}+1}}}{\Delta \sigma} .
$$

Similarly, the instantaneous rate of change of the reflected radiance in the tilt direction of $\Delta \tau$ is approximated by the quantity

$$
\frac{\frac{p \cos (\tau+\Delta r) \sin \sigma+q \sin (\tau+\Delta r) \sin \sigma+\cos \sigma}{\sqrt{p^{2}+q^{2}+1}}-\frac{p \cos r \sin \sigma+q \sin r \sin \sigma+\cos \sigma}{\sqrt{p^{2}+q^{2}+1}}}{\Delta r} .
$$

Consider the expression in equation 1 as a function of the variables $\sigma$ and $\tau$ where $\mathrm{p}$ and $\mathrm{q}$ are fixed. That is, the reflected radiance at a specific point on an object surface is being viewed as a function of the 
incident orientation of the light source. Mathematically speaking the expressions in 2 and 3 represent an approximation to the directional derivatives of the function in equation 1 in the unit vector directions $\partial_{\sigma}$ and $\partial_{\tau}$ respectively. The vectors $\partial_{\sigma}$ and $\partial_{\tau}$ are unit vectors which span the tangent space at a point on the two dimensional manifold of the coordinates $(\sigma, \tau)$. These directional derivatives are computed as follows:

and hence

$$
\partial_{\sigma} R(\sigma, \tau)=\frac{\partial R}{\partial \sigma}, \quad \partial_{\tau} R(\sigma, \tau)=\frac{\partial R}{\partial \tau}
$$

$$
\begin{gathered}
\partial_{\sigma} R(\sigma, \tau)=\frac{p \cos \tau \cos \sigma+q \sin \tau \cos \sigma-\sin \sigma}{\sqrt{p^{2}+q^{2}+1}} \\
\partial_{\tau} R(\sigma, \tau)=\frac{-p \sin \tau \sin \sigma+q \cos \tau \sin \sigma}{\sqrt{p^{2}+q^{2}+1}}
\end{gathered}
$$

It is possible to take different linear combinations of directional derivatives as follows

$$
\left(a \partial_{\sigma}+b \partial_{\tau}\right) R(\sigma, \tau)=a \frac{\partial R}{\partial \sigma}+b \frac{\partial R}{\partial \tau}
$$

where $a$ and $b$ are any real values. This property of directional derivatives can be put to good use in experimental implementation to reduce errors. Moving the illuminator in certain specified directions can create nearly perpendicular intersections of curves in gradient space.

Note that the function in equations 4 and 5 has the same form as in equation 1 . Any of these functions set equal to a constant value measured from experiment would generate a conic section in gradient space.

The equations 4 and 5 define Lambertian photometric flow fields in positive $\sigma$ and $\tau$ respectively. These can be measured empirically from the expressions in 2 and 3 where the denominator should be the angular variation measured in radians. In a sense Lambertian photometric flow fields are like different Lambertian reflectance functions except that equations 4 and 5 take on negative values as well as positive values.

Figure 2 shows the rendering of a Lambertian sphere using a point light source at incident orientation $\sigma=45$ degrees and $\tau=45$ degrees. Figures 3 show a computer simulation of photometric flow fields on the Lambertian sphere, in figure 2 , from $t$ wo independent angular variations of the light source. Figure $3 \mathrm{a}$ shows the instantaneous rate of change of image irradiance as the light source orientation changes in positive $\sigma$. Figure $3 \mathrm{~b}$ shows the instantaneous rate of change of image irradiance as the light source orientation changes in positive $\tau$. For figure $3 \mathrm{a}$ intensity values northeast of the dark band represent positive reflected radiance changes, while intensity values southwest of the dark band represent negative changes. For figure $3 \mathrm{~b}$ the positive reflected radiance changes are northwest of the dark band, and negative reflected radiance changes are to the southeast. Figures $\mathbf{4 a}$ and $\mathbf{4 b}$ show the corresponding relative percentage changes in the reflected radiance produced by the changes represented in figures $3 \mathrm{a}$ and $3 \mathrm{~b}$ respectively. It should be noted that the image intensity values in figures 3 and 4 are relative values. The maximum change over all points on the sphere is set to value 255 with all other values relative to this maximum intensity value. Clearly, maximum values for the photometric flow fields occur at local surface orientations generally far away from the incident orientation of the point light source.

Of immediate notice are the dark bands that are present on the sphere in figures 3 and 4 . The intensity values on these bands are very close to zero, and are actually zero along the medial axis of the bands. The medial axes of the dark bands in figures 3 and 4 are examples of isoflow curves on the surface of the sphere 
along which the photometric flow fields are some constant value. In the next section it will be shown that isoflow curves determine local surface orientation constraints. A very simple orientation constraint is that generated by the zero valued isoflow curve which is the medial axis of the dark band in figure $3 \mathrm{a}$. For any points along this isoflow curve the tilt $r$ of the local surface orientation is equal to the tilt value of the incident orientation of the light source. Most orientation constraints are much more complicated than this.

In a practical experiment where photometric flow fields would be determined from empirical measurement of reflected radiance from a CCD camera, there are two major sources of errors to consider. The first is the measured reflected radiance itself. Enough angular variation in $\sigma$ and $\tau$ needs to be made so that the change in reflected radiance should significantly exceed the repeatability of the photoresponse of pixel sensors. This amount is dictated by the signal to noise ratio of the camera being used. Also the output of light sources is not constant over time. The measurements of reflected radiance should be made as quickly as possible between angular variations. The second major source of errors is in the approximation of equations 4 and 5 respectively by expressions 2 and 3 .

\section{SURFACE ORIENTATION FROM PHOTOMETRIC FLOW FIELDS}

This section will simulate by computer the quantitative derivation of local surface orientation, with the aid of constraints provided from a pair of photometric flow fields. As was seen in the last section, constraints on local surface orientation can be obtained from isoflow curves which are derived by setting equations 4 and 5 to constant values. Examples of isoflow curves in gradient space are shown in figures $5 \mathrm{a}$ and $5 \mathrm{~b}$ with respect to photometric flow fields derived from positive variations in $\sigma$ and $\tau$ respectively. The incident orientation of the light source used is the same as for the simulations in figures 3 and 4 with $\sigma=\tau=45$ degrees.

The isoflow curves in gradient space depicted in figures $5 \mathrm{a}$ and $5 \mathrm{~b}$ are conic sections of varying eccentricity. The only difference between these isoflow curves and a Lambertian reflectance map depicting isoreflectance curves is that the constant value of photometric flow that an isoflow curve represents can be negative. At a point on a smooth object surface, local surface orientation is constrained by empirically determined values of photometric flow in positive sigma and positive tau which determine the intersection of two isoflow curves in gradient space. Sometimes this results in a unique orientation point, but the rest of the time local surface orientation is only constrained to be at two distinct points. It is not possible to breakup this two point a mbiguity by using another photometric flow field obtained by moving the light source in another direction. The reason is that for small angular motions of the light source this direction is simply a linear combination of the motions in $\sigma$ and in $\tau$. The isoflow line thus obtained would therefore pass through the same two points contraining orientation.

A third constraint curve on local surface orientation in gradient space can be obtained from the isoreflectance curve corresponding to the measured reflected radiance at the point in question. This is simulated in figure 6 for a point on a smooth surface with local surface orientation $(-1.0,0.5)$ in gradient space coordinates. The isoflow curves in figure 6 can be identified by observing the curves in figures $5 \mathrm{a}$ and $5 \mathrm{~b}$ in the same region of gradient space.

Figure 7 depicts the intersection of three isoreflectance curves making small angular variations in the illuminator of 5 degrees in $\sigma$ and $\tau$. This would be the result of using conventional photometric stereo with very small displacements in the incident orientation of the light source. Under ideal circumstances free of 
measurement errors this would work fine. Figures $8 \mathrm{a}$ and $8 \mathrm{~b}$ show, in the presence of measurement errors, the comparison of measuring local surface orientation using photometric flow fields with doing this measurement using conventional photometric stereo. These are simulations of worst case errors in the presence of $\pm 5 \%$ error in measured reflected radiance. The simulated empirical values for the photometric flow fields are derived by taking the ratios in equations 1 and 2 for 5 degree variations in $\sigma$ and $\tau$ respectively. Even in the presence of approximation error the centroid of the three two-way intersection points in figure 8 a produce a measurement error of about 5 degrees, while the measurement error in figure $8 \mathrm{~b}$ is well over 20 degrees.

\section{ILLUMINATOR ORIENTATION FROM PHOTOMETRIC FLOW FIELDS}

Because photometric flow fields are determined locally there exists a duality between the determination of local surface orientation using the known incident orientation of a light source and the determination of the incident orientation of a light source using the known local surface orientation at a point on a Lambertian surface. This is illustrated by observing equations 4 and 5 and the roles that $(p, q)$ and $(\sigma, \tau)$ play as variables and known constant values. In the last section, local surface orientation was determined from isoflow curves assuming that $(\sigma, \tau)$ were known and constant and $(p, q)$ were variables to be solved. But another interpretation of equations 4 and 5 can be that $(p, q)$ are known, meaning that something like a Lambertian calibration block is used, and the illuminator orientation $(\sigma, \tau)$ are variables to be solved. This is an advantage of using photometric flow fields over using conventional photometric stereo since the empirically determined values for the photometric flow fields along with the reflected radiance value all correspond to exactly the same incident orientation of the illuminator.

To demonstrate this duality, a simulation will be performed which reverses the knowns and unknowns of the simulation performed in the last section. Starting with the known orientation value $(p, q)=(-1.0,0.5)$, the simulation in this section will use isoflow curves to determine the unknown incident orientation of the light source (which in actuality is $(\sigma, \tau)=(45,45)$ ). To generate curves in gradient space, the variables once again should be in terms of $(p, q)$. To do this the variables $(p, q)$ in equations 4 and 5 will be held fixed at known values $\left(p_{0}, q_{0}\right)$ and the angular representation $(\sigma, \tau)$ will be converted to gradient space representation according to

$$
\begin{array}{cc}
\cos \sigma=\frac{1}{\sqrt{p^{2}+q^{2}+1}}, \quad \sin \sigma=\frac{\sqrt{p^{2}+q^{2}}}{\sqrt{p^{2}+q^{2}+1}} \\
\cos \tau=\frac{p}{\sqrt{p^{2}+q^{2}}}, \quad \sin \tau=\frac{q}{\sqrt{p^{2}+q^{2}}}
\end{array}
$$

whereupon equations 4 and 5 are equivalently

$$
\begin{gathered}
\partial_{\sigma} R(\sigma, \tau)=\frac{p_{0} p+q_{0} q-\left(p^{2}+q^{2}\right)}{\sqrt{p_{0}^{2}+q_{0}^{2}+1} \sqrt{p^{2}+q^{2}} \sqrt{p^{2}+q^{2}+1}} \\
\partial_{\tau} R(\sigma, \tau)=\frac{-p_{0} q+q_{0} p}{\sqrt{p_{0}^{2}+q_{0}^{2}+1} \sqrt{p^{2}+q^{2}+1}}
\end{gathered}
$$

respectively. 
Isoflow curves from variations in $\tau$ are once again conic sections in gradient space as can be seen by the form of equation 7. However isoflow curves from variations in $\sigma$ are more complicated. A graphical depiction of isoflow curves produced from setting equation 6 to different constant values is given in figure 9 . In figure 10 is the determination of the incident orientation of the illuminator from two isoflow curves from variations in $\sigma$ and $\tau$ respectively, and the isoreflectance curve corresponding to the reflected radiance value at the point on the smooth surface.

\section{CURVATURE FROM PHOTOMETRIC FLOW FIELDS}

In [Woodham 1978] and [Woodham 1979] a method is presented which determines viewer-centered curvature constraints from shading information. Starting with the image irradiance equation

$$
I(x, y)=R(p, q)
$$

an application of the chain rule for derivatives yields the matrix equation

$$
\left(\begin{array}{l}
\partial I / \partial x \\
\partial I / \partial y
\end{array}\right)=\left(\begin{array}{ll}
\partial p / \partial x & \partial p / \partial y \\
\partial q / \partial x & \partial q / \partial y
\end{array}\right)\left(\begin{array}{l}
\partial R / \partial p \\
\partial R / \partial q
\end{array}\right)
$$

The $2 \times 2$ matrix in equation 8 represents the Jacobian of the transformation from image coordinates to local surface orientation normals for a given surface. These normals are represented in gradient space coordinates. Hence when this matrix multiplies an infinitesimal vector change in image coordinates, the resulting vector represents the infinitesimal vector change in the local surface normal of the surface at that point. That is,

$$
\left(\begin{array}{l}
d p \\
d q
\end{array}\right)=\left(\begin{array}{ll}
\partial p / \partial x & \partial p / \partial y \\
\partial q / \partial x & \partial q / \partial y
\end{array}\right)\left(\begin{array}{c}
d x \\
d y
\end{array}\right)
$$

Hence this Jacobian transformation is termed the viewer-centered curvature matrix with respect to image coordinates. On a smooth surface which is parametrized by height above the image plane as $(x, y, f(x, y))$, the viewer-centered curvature matrix is equivalently the Hessian matrix

$$
\left(\begin{array}{ll}
\partial^{2} f / \partial x^{2} & \partial^{2} f / \partial y^{2} \\
\partial^{2} f / \partial x^{2} & \partial^{2} f / \partial y^{2}
\end{array}\right)
$$

This utilizes the standard definition of gradient space coordinates as

$$
p=\partial f / \partial x \quad q=\partial f / \partial y .
$$

To solve for the three components of the viewer-centered curvature matrix in equation 8 at a point on a smooth surface, the local surface orientation is required to be known in order to compute the gradient of the reflectance map $R(p, q)$. Assuming this is known. equation 8 represents an underconstrained pair of linear equations in three unknowns. In [Woodham 1978] and [Woodham 1979] it is proposed that the underconstrained nature of equation 8 can be ameliorated by solving for certain classes of smooth surfaces. These include developable surfaces for which the determinant of the viewer-centered curvature is always zero, and convex surfaces for which this determinant is greater than zero. 
In [Wolff 1987] it is proposed that the viewer-centered curvature matrix can be solved for arbitrary smooth surfaces by combining curvature from shading with photometric stereo. From two additional light source orientations, the two additional matrix equations

$$
\begin{aligned}
& \left(\begin{array}{l}
\partial I / \partial x \\
\partial I / \partial y
\end{array}\right)=\left(\begin{array}{ll}
\partial p / \partial x & \partial p / \partial y \\
\partial q / \partial x & \partial q / \partial y
\end{array}\right)\left(\begin{array}{l}
\partial R^{\prime} / \partial p \\
\partial R^{\prime} / \partial q
\end{array}\right) . \\
& \left(\begin{array}{l}
\partial I / \partial x \\
\partial I / \partial y
\end{array}\right)=\left(\begin{array}{ll}
\partial p / \partial x & \partial p / \partial y \\
\partial q / \partial x & \partial q / \partial y
\end{array}\right)\left(\begin{array}{l}
\partial R^{\prime \prime} / \partial p \\
\partial R^{\prime \prime} / \partial q
\end{array}\right) .
\end{aligned}
$$

solve for the exact same viewer-centered curvature matrix. This not only obviates the need for assumed auxiliary constraints on the viewer-centered curvature matrix, but in fact overconstrains the equations for better recovery in the presence of measurement error.

The shading information provided by photometric flow fields can equivalently be used to determine the viewer-centered curvature matrix for a smooth surface. That is the reflectance maps $R^{\prime}(p, q)$ and $R^{\prime \prime}(p, q)$ in equations 9 and 10 can be replaced by $\partial_{\sigma} R(p, q)$ and $\partial_{\tau} R(p, q)$ from equations 4 and 5 respectively. Note however that the image intensity gradients are now photometric flow field gradients in the image plane. In fact two matrix equations resulting from two photometric flow fields obtained from linearly independent angular variations are enough to overconstrain the determination of the three components for the viewercentered curvature matrix. The solution can be further overconstrained by the use of the original reflectance map using the incident orientation of the light source.

\section{CONCLUSION}

It has been demonstrated that shape characteristics for smooth surfaces such as local surface orientation and curvature can be derived by examining the instantaneous rate of change in the reflected radiance at points on a smooth surface with respect to angular change in illumination geometry. This rate of change in reflected radiance is determined by the directional derivative of the reflectance function in the angular direction in which the incident orientation of the light source varies. Because the incident orientation of a light source has two angular degrees of freedom, two independent directional derivatives exist giving rise to two independent equations at a point involving local surface orientation.

The approximation to a directional derivative of the reflectance function, in a given direction, is derived at each pixel from two slightly displaced incident orientations of the illuminating light source. This photometric approximation at each pixel in an image corresponding to an illuminated object point constitutes what is termed a photometric flow field. At each pixel, the measured value of a photometric flow field constrains local surface orientation at the corresponding object point according to the locus of an isoflow curve in gradient space. This is in the same flavor as constraining local surface orientation from photometric stereo using isoreflectance curves. It was shown that curvature at points on a smooth surface can be obtained from the gradients of photometric fow fields in the image plane.

Because isoflow curves constraining local surface orientation at an object point correspond to the same incident illuminator orientation it was shown that there is a duality between using photometric flow fields to obtain local surface orientation from known incident illuminator orientation and obtaining incident illuminator orientation from known local surface orientation. This duality does not exist for photometric stereo. 


\section{ACKNOWLEDGEMENTS}

The author is grateful to David Kurlander for rendering the images of the Lambertian sphere and the resulting photometric flow fields.

\section{References}

[Horn 1975]

[Horn 1977]

[Ikeuchi 1981]

[Ikeuchi et al. 1986]

[Klinker, Shafer and Kanade 1987]

[Pentland 1984]

[Shafer 1985]

[Wolff 1987]

[Wolff 1988]

[Woodham 1978]

[Woodham 1979]
Horn, B.K.P., Obtaining shape from shading information, in The Psychology of Computer Vision, P.H. Winston (ed.), McGraw-Hill, pp.115155, New York, 1975.

Horn, B.K.P., Understanding image intensities, Artificial Intelligence, 8, 1977, pp.1-31.

Ikeuchi, K., Determining surface orientations of specular surfaces by using the photometric stereo method, PAMI, Vol. 3, No. 6, pp.661-669, November 1981.

Ikeuchi, K., Nishihara, H.K., Horn, B.K., et al..., Determining Grasp Configurations using photometric stereo and the PRISM binocular stereo system, The International Journal of Robotics, Vol. 5, No. 1, pp.46-65, Spring 1986.

Klinker, G.J., Shafer, S.A. and Kanade, T., Using A Color Reflection Model to Separate Highlights From Object Color, Proceedings of the First International Conference on Computer Vision, pp. 145-150, 1987.

Pentland, A.P., Local Shading Analysis, IEEE trans. on Pattern Analysis and Machine Intelligence, Vol. 6, No. 2, pp. 170-187, March 1984.

Shafer, S.A., Using Color To Separate Reflection Components, COLOR research and application, 10(4), pp.210-218, Winter 1985.

Wolff, L.B., Curvature and contour from photometric stereo. DARPA image understanding workshop, 1987, pp. 821-824.

Wolff, L.B., Segmentation of specular highlights from object surfaces, SPIE Cambridge 1988, Optics, Illumination, and Image Sensing For Machine Vision III.

Woodham, R.J., Refiectance map techniques for analyzing surface defects in metal castings. MIT AI Lab Tech Report AI-TR-457, June 1978.

Woodham, R.J., Relating properties of surface curvature to image intensity, IJCAI 1979, pp. 971-977. 


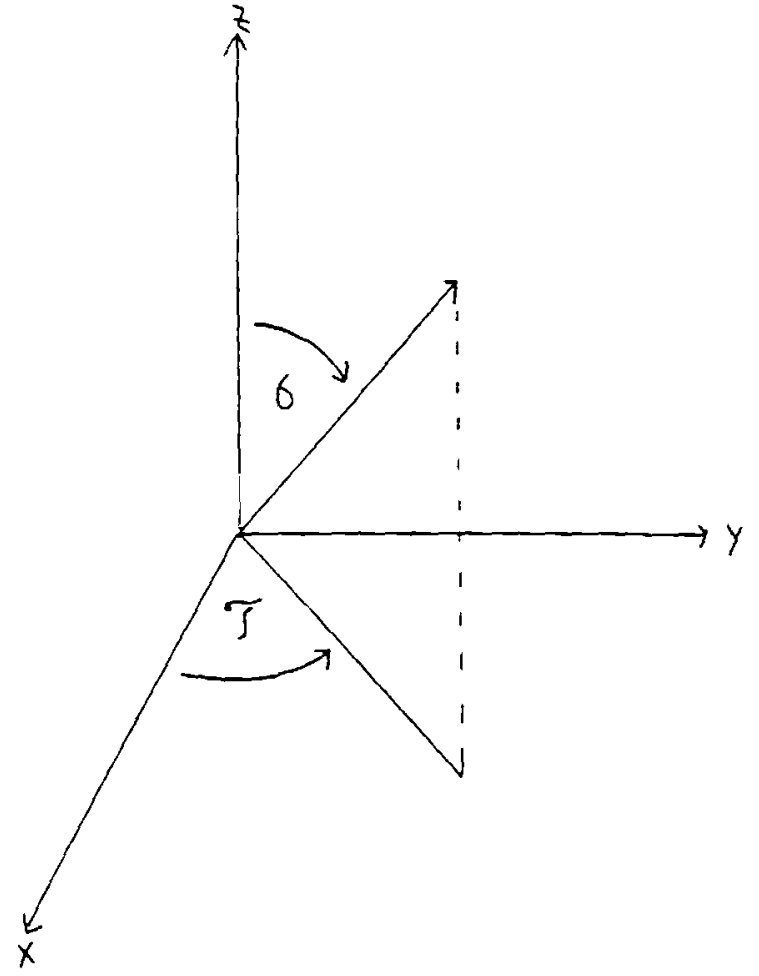

FIGURE 1

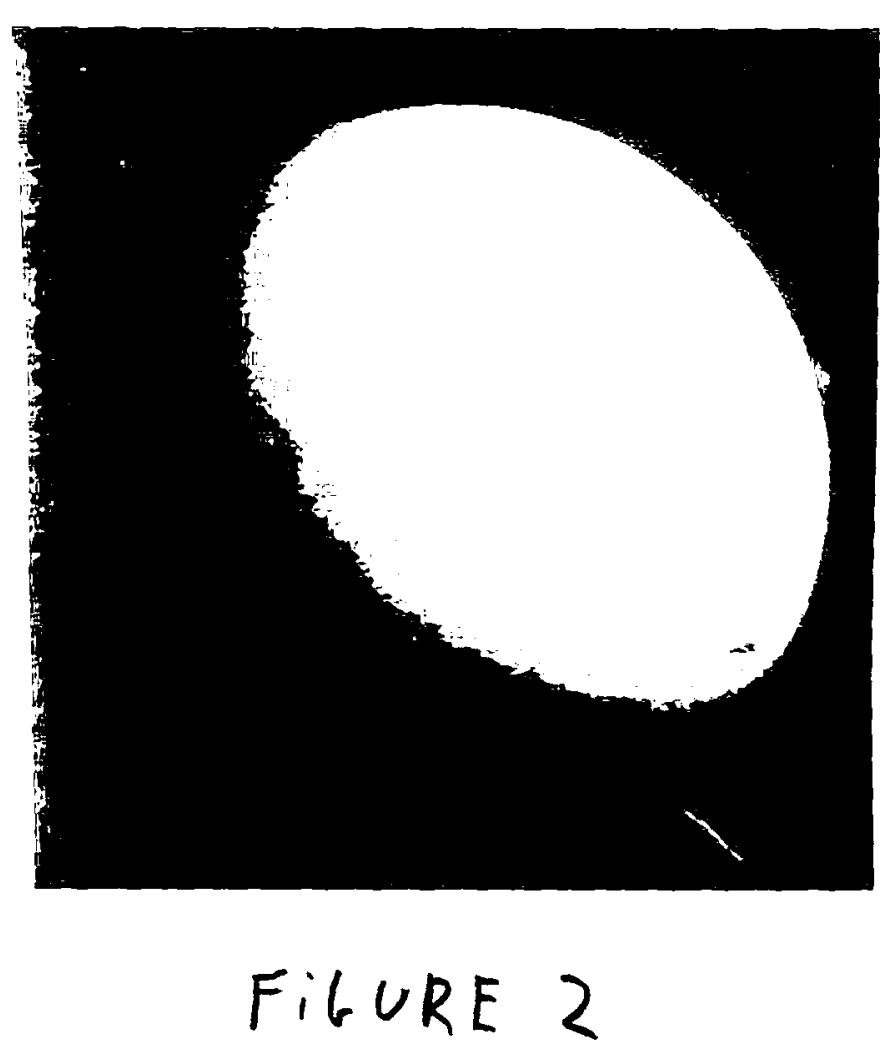

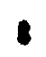



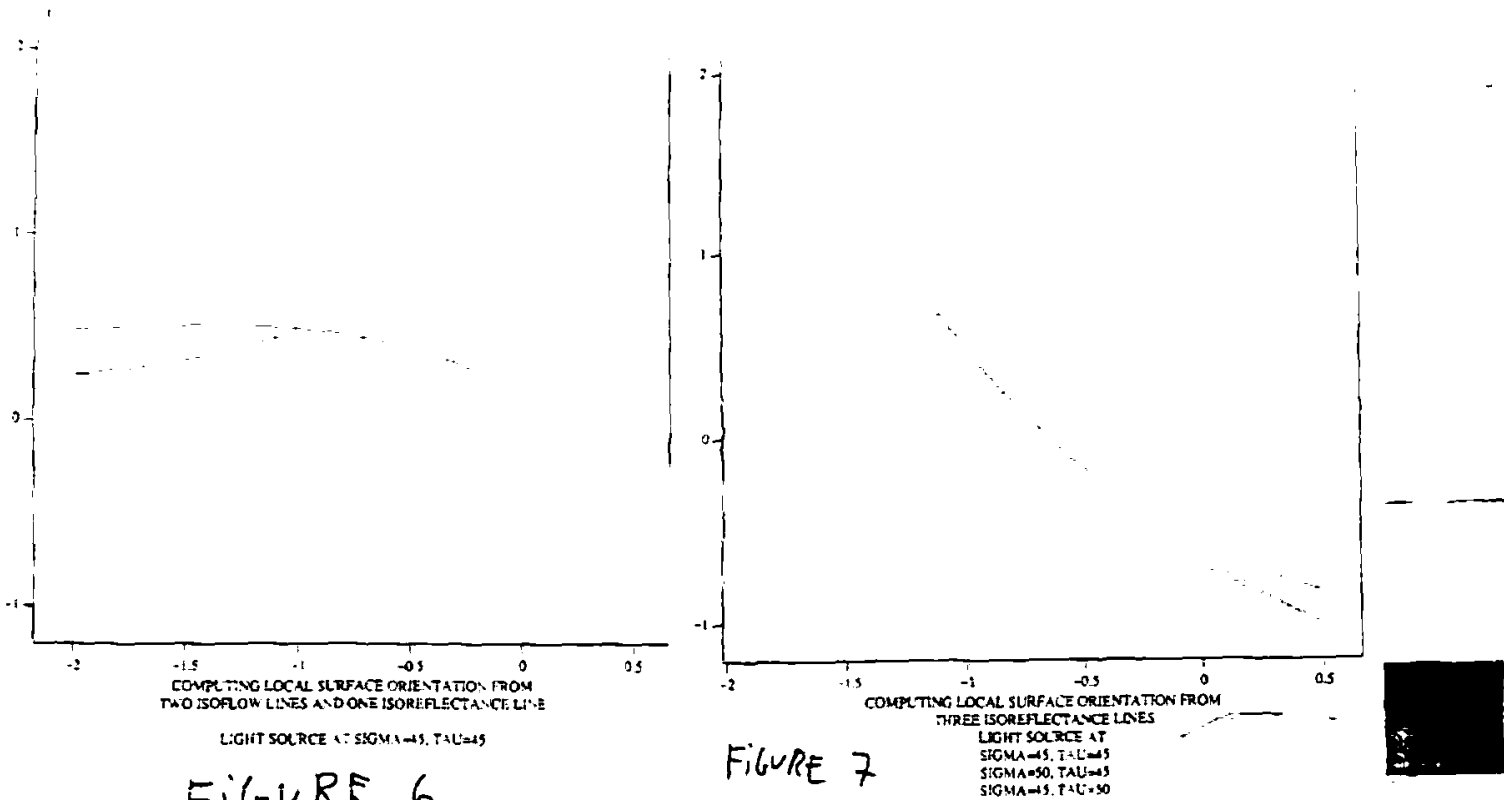

\section{FilURE 6}

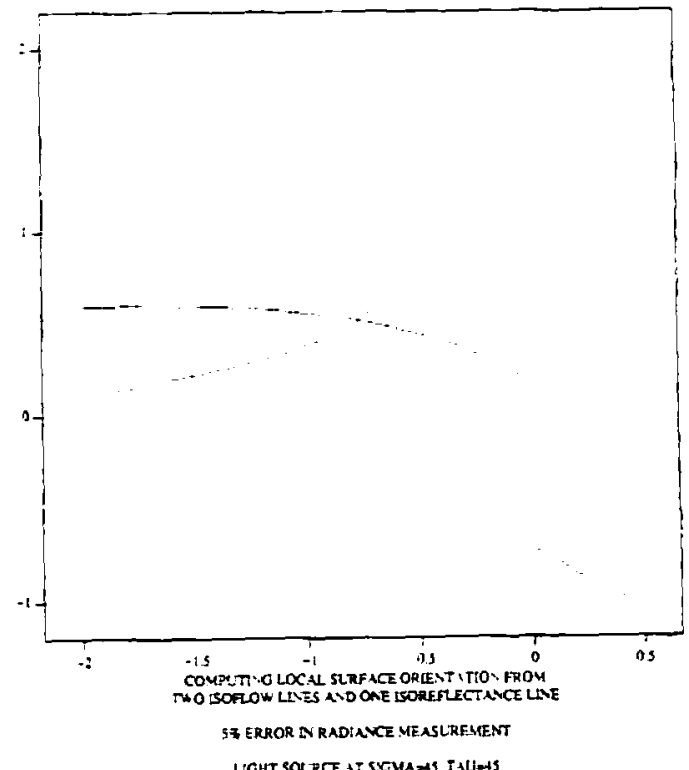

FilURE $8 a$

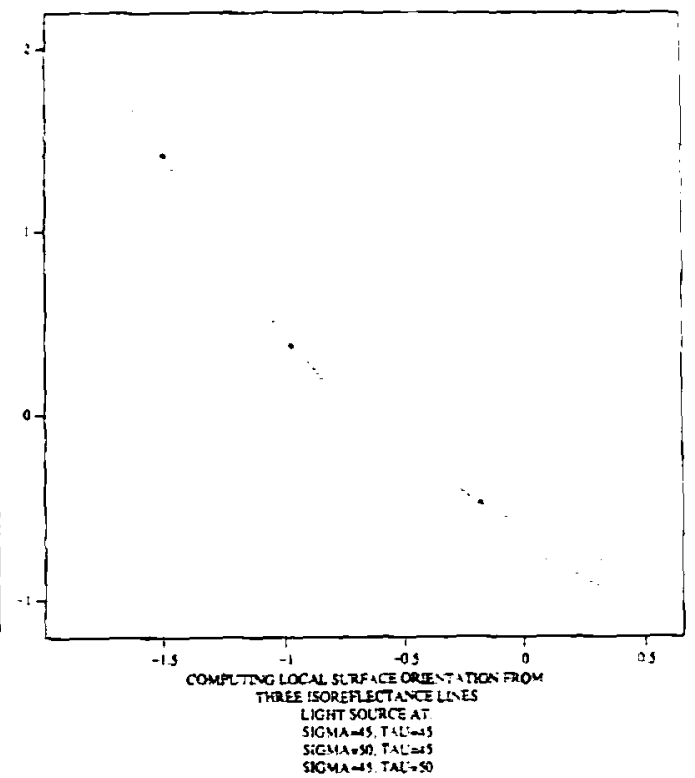

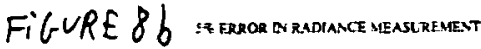

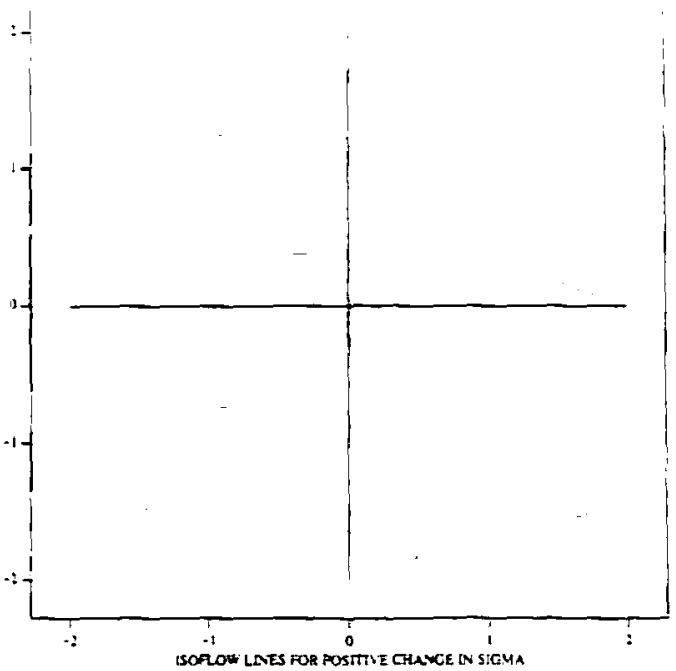

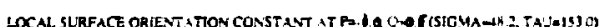

FIGURE 9

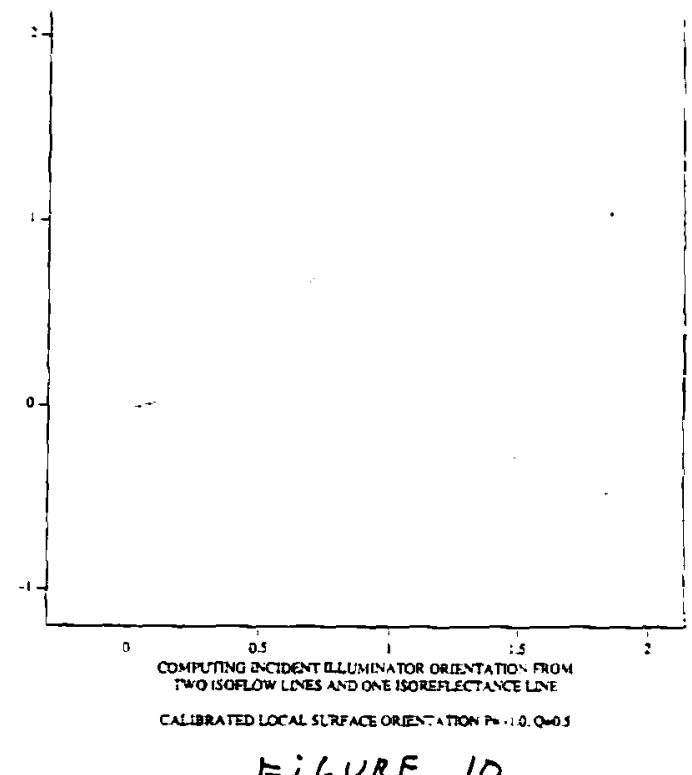

